\title{
Learning Open-World Object Proposals without Learning to Classify
}

\author{
Dahun Kim \\ KAIST
}

\author{
Tsung-Yi Lin \\ Google Brain
}

\author{
Anelia Angelova \\ Google Brain
}

\author{
In So Kweon \\ KAIST
}

\author{
Weicheng Kuo \\ Google Brain
}

\begin{abstract}
Object proposals have become an integral preprocessing steps of many vision pipelines including $o b$ ject detection, weakly supervised detection, object discovery, tracking, etc. Compared to the learning-free methods, learning-based proposals have become popular recently due to the growing interest in object detection. The common paradigm is to learn object proposals from data labeled with a set of object regions and their corresponding categories. However, this approach often struggles with novel objects in the open world that are absent in the training set. In this paper, we identify that the problem is that the binary classifiers in existing proposal methods tend to overfit to the training categories. Therefore, we propose a classification-free Object Localization Network (OLN) which estimates the objectness of each region purely by how well the location and shape of a region overlap with any ground-truth object (e.g., centerness and IoU). This simple strategy learns generalizable objectness and outperforms existing proposals on cross-category generalization on COCO, as well as cross-dataset evaluation on RoboNet, Object365, and EpicKitchens. Finally, we demonstrate the merit of OLN for long-tail object detection on large vocabulary dataset, LVIS, where we notice clear improvement in rare and common categories.
\end{abstract}

\section{Introduction}

Object proposals are a set of regions or bounding boxes that contain objects with high likelihood [33, 41, 42, 45, 59-61, 74]. They have become the integral pre-processing steps for many computer vision systems, including object detection [33,41, 42, 45, 59, 74], segmentation [3, 8, 13], object discovery $[12,16,47]$, weakly supervised object detection [5, 21, 53], visual tracking [35, 63], content-aware retargeting [51], etc. Due to the success of object detection, the recent trend in object proposal research has shifted from object discovery to detection. While the goal of object discovery proposals is to propose any objects in the image, the goal of detection proposals is to propose only the la-
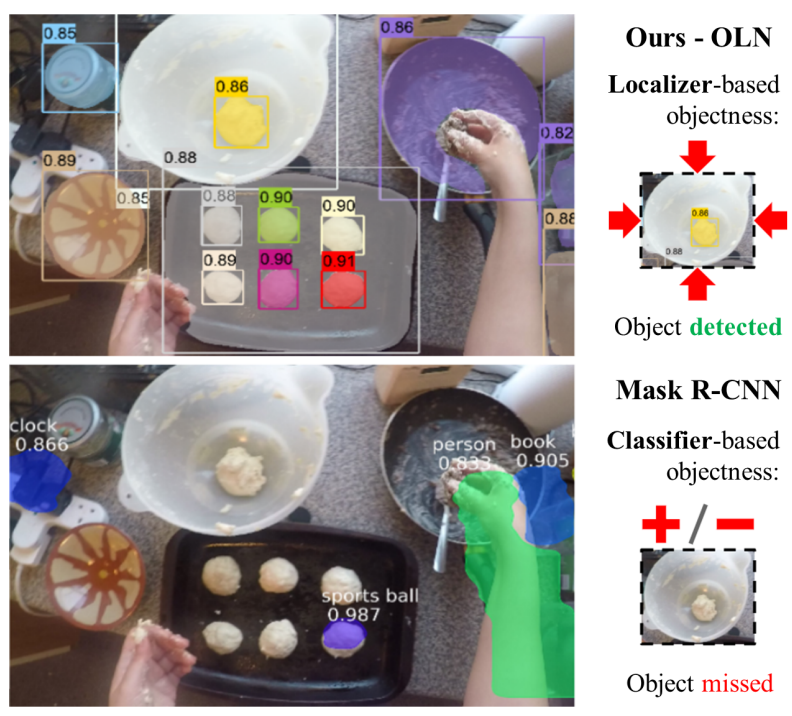

Mask R-CNN

Classifier-based objectness:

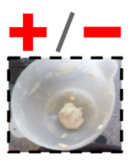

Object missed

Figure 1: Humans can recognize novel objects in this image despite having never seen them before. "Is it possible to learn open-world (novel) object proposals?" In this paper we propose Object Localization Network (OLN) that learns localization cues instead of foreground vs background classification. Only trained on COCO [38], OLN is able to propose many novel objects (top) missed by Mask R-CNN (bottom) on an out-of-sample frame in an ego-centric video.

beled categories for downstream classifier. Learning-based proposals are popular detection proposals because of simplicity and shared computation with downstream detection. However, unlike their learning-free counterparts [42,59,74], these methods tend to overfit to annotated categories and struggle with novel objects [33, 41, 64]. We want to ask, is it possible to combine the best of both worlds and "learn open-world (novel) object proposals?" This could potentially unlock learning-based proposals for promising applications including open-world detection [30] / segmentation [66], robot grasping [15], egocentric video understanding [14], and large vocabulary detection [24].

Given a set of object annotations, we want to learn what general objects look like and propose highly dissim- 
ilar object candidates from unseen categories and new data sources. This matches the ability of human to detect novel objects in new environments without naming their categories e.g. a piece of obstacle on the road, a novel product on the shelf. Our main insight is that the classifiers in existing object proposers $[33,41,60]$ or class agnostic detectors $[45,56]$ impedes such generalization, because the model tends to overfit to labeled objects and treat the unlabeled objects in the training set as background. We propose Object Localization Network (OLN), which learns to detect objects by predicting how well a region is localized instead of performing foreground-background classification. This simple idea allows the model to learn stronger objectness cues. To our best knowledge, we are the first to demonstrate the value of learning pure localization-based objectness for proposing novel objects, although the idea of incorporating the localization quality estimation has been proposed by others in the standard fixed-category detection setting [28, 29, 56, 69]. We show that a classifier-free object proposer is key to achieve optimal cross-category and cross-dataset generalization, which is an important design difference to existing proposers or class agnostic detectors.

We study the efficacy of OLN on the COCO cross category setting following existing works $[33,41,64]$. Despite the simplicity, OLN outperforms the state-of-the-art by $\mathbf{+ 3 . 3}$ AUC (+5.0 AR@10,+5.1 AR@100) on novel categories. Our ablation studies confirm that the use of foreground-vsbackground classifier hurts, and that localization helps. In addition, we study cross-dataset generalization from $\mathrm{COCO}$ to RoboNet [15], Objects365 [48], and EpicKitchens [14]. We chose RoboNet because it contains a wide range of novel objects common in robotics grasping application and the bin environment permits more reliable exhaustive annotation for proper evaluation [9]. On RoboNet, OLN performs exhaustive, class-agnostic object detection and outperforms the standard approach by $+\mathbf{1 3} \sim 16 \mathrm{AP}$, whereas on Objects365 OLN has +4 AR@10 and +8 AR@100 over the standard approach. Qualitative visualization on EpicKitchens [14] further shows that OLN outperforms the standard approach in detecting a variety of novel objects. Last but not least, we apply OLN as a drop-in replacement for RPN [45] on LVIS long tail detection [24] and observe a gain of +1.4 AP, mostly attributed to the rare (+3.4 APr) and common categories $(+1.8 \mathrm{APc})$. This shows OLN is able to capture the long tail in large vocabulary detection.

It is worth noting that estimating localization quality is not new in the standard detection, but they are always used alongside classification and validated on seen categories only, e.g. FCOS [56]. To our knowledge, we are the first to explore the use of localization cues independent of classification for object proposals. This discovery helps us obtain notable gains on COCO and generalize to many dissimilar datasets better than existing method.
Our contributions can be summarized as follows:

- To our knowledge, we are the first to show the value of pure localization-based objectness learning for novel object proposals, and propose a simple-yet-effective classifier-free Object Localization Network (OLN).

- Our approach outperforms state-of-the-art methods on cross-category setting on COCO and improves crossdataset settings on RoboNet and Object365, long-tail detection (LVIS) and egocentric videos (EpicKitchens) over the standard approach.

- We carefully annotated the RoboNet dataset for the presence of all objects in an exhaustive fashion. We perform open-world class-agnostic object detection, and evaluate the Average Precision, which also improves existing AR-based evaluation of proposals on partially-annotated data.

- Extensive ablation and analysis on OLN modeling choices reveal the benefits of each localization cue and the overfitting of existing classifier-based methods.

\section{Related Work}

Below we discuss existing efforts to improve proposal and detection quality, and works that scale detection to more visual categories.

Object proposal. In early works, the emphasis was on category-independent object proposals [2, 4, 18, 39, 74], where the goal is to identify instances of all objects in the image irrespective of their category. These works utilize hand-crafted heuristics to capture the notion of general objects [59,74], i.e., color contrast, edge.

Recently, learning-based proposals [23, 33, 36, 41, 45] have demonstrated better performance than classical approaches in both precision and recall, and are an important part of two-stage detectors. A representative example is region proposal network (RPN) [45] which identifies a set of regions in a given image that could contain objects, which are then used by a downstream detector module to localize and classify objects. A number of follow-up works $[23,60,61,72]$ have been proposed to improve the quality of such region proposals and reduce their number in order to speed up the final detection task. In fact, these proposal modules are trained end-to-end with the detector module, where the notion of objectness is defined by a set of training categories in the dataset. Despite their progress in detecting objects of the known, supervised categories, learning-based proposals still struggle on novel objects.

More closely related to our work are studies on generalization of object proposals onto unseen classes. Chavali et al. [9] demonstrate the standard proposal evaluation is problematic or "gameable" in evaluating category-independence 
of object proposal because detection of unknown object classes is explicitly penalized in the benchmark protocol. Wang et al. [64] study the generalization from a dataset standpoint and demonstrate the impact of visual diversity and label granularity of training dataset on the generalization of object proposers. In contrast, we focus on the modeling choices in designing an object proposer that can generalize to novel categories and new datasets.

Multi-class detection. A number of efforts have been made to scale up the number of classes for detection by transferring commonalities between object categories with varying degrees and quality of supervision.

Weakly-supervised approaches $[5,7,17,54,62]$ aim to utilize abundant image-level labels and leverage classagnostic box proposals to build detectors. Approaches under semi-supervised setup [27,44,49,50,55, 58, 73] employ the weak image-level labels for novel classes as well as boxlevel labels for base classes. For example, YOLO-9000 [44] and R-FCN-3000 [49] concurrently train on box-level and image-level data to scale up the detector's class coverage. Knowledge transfer-based methods learn to transfer the proposals from base to novel classes based on their similarity on semantic hierarchy. This line of research is also related to few-shot $[10,31,67,68,71]$ and zero-shot detection $[6,20,43,70]$ methods, which attempt to detect novel classes given only a few samples or class descriptions.

In contrast to the above class-specific detectors, our goal is to go beyond the concept of category and detect all objects in a category-independent manner (without classification). Even though the multi-class detectors enumerate many categories, they still fail to generalize for unseen/unknown object categories.

Dataset efforts for detection at scale. Existing datasets focus on single-dataset settings [19, 24, 34, 38, 48]. Models trained on one dataset are only evaluated on the same dataset. Recently the Robust Vision Challenge [1] is a first step towards cross-dataset benchmark. We hypothesize that learning objectness on one dataset should also transfer to another dataset, as the saliency cues tend to be more generalizable than class-specific information. Therefore, in this work we study the generalization setting of training on COCO, then testing on other datasets: RoboNet [15], Objects365 [48], EpicKitchens [14], and LVIS [24].

Localization cues for object detection. Researchers have studied many ways to improve the localization quality in object detections by learning centerness [56], iterative proposal refinement [60], or box/mask IoU prediction [28, 29, 57]. These approaches have demonstrated meaningful improvement in the standard object detection task. However, it remains an open question whether these methods can transfer to novel categories. In addition, most of these works use these localization cues alongside classifier

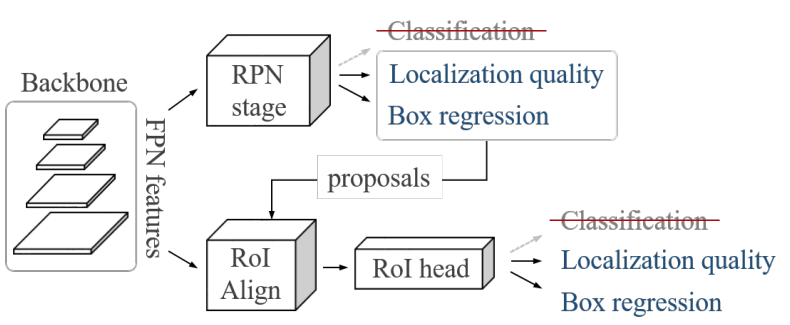

Figure 2: Overview of Object Localization Network (OLN). We replace the classifier head which is common in most object proposal methods with localization quality estimators, e.g. centerness and IoU. This applies to the anchors at whole-image level and to each region of interest (RoI).

outputs. It is unclear whether these cues on their own can effectively distinguish objects from background.

\section{Proposed Method}

\subsection{Baselines}

Before we describe OLN in section 3.2 and 3.3, we would like to define the baselines which can address the same unseen category generalization problem. Region proposal network (RPN) [45, 60,61] is the most common family of approaches of objectness learning in object detection. By design, RPNs aim to propose all objects in the image regardless of their categories, but in practice they often struggle when encountered with novel objects in the open world. Another family of baseline is to train existing object detectors in a class-agnostic fashion by treating all annotated categories as one foreground category. As OLN is built upon RPN and Faster R-CNN, we use both as strong baselines throughout the paper. Moreover, we provide comparisons with different state-of-the-art models in region proposal $[45,60,61]$ and object detection $[25,45,56]$. As seen in our experiments later, OLN outperforms all of them in various generalization scenarios.

\subsection{Pure localization-based objectness}

In the context of learning-based object proposals, "object" is defined as a set of annotated categories, and the learning of objectness is cast as a binary classification task: whether or not a region belongs to the union of the predefined categories. However, our main insight is that such discriminative learning of the foreground-vs-background problem impedes generalization because the model learns to classify the unlabeled/unknown objects as background. To address this problem, we propose a non-discriminative and classification-free notion of objectness.

The classification view of "objectness" is to ask "how much does this region look like a foreground object?" From a localization standpoint, we want to ask instead "how well 
does this region overlap with any ground-truth object?". Our intuition is that every object can be characterized by its location and shape, regardless of its category. OLN leverages these geometric cues to capture the objectness of a proposed region. We demonstrate that the learnt objectness cues based on the localization (location and shape) quality can collectively improve the generalization of object proposals beyond the labeled categories and data sources. We adopt centerness [56] and IoU score [29] for location and shape quality measures respectively, while not restricting other choices such as Dice coefficient [40] and generalizedIoU [46].

The idea of incorporating localization quality is not totally new in object detection. Several works [28, 29, 56, 57] recalibrate the final detection confidence by using both localization and classification subnets. Note that, however, their localization cues are thoroughly an auxiliary to the classifiers and are devised for within-category detection. In contrast, we demonstrate that a pure localization-based objectness is the key to generalize beyond category and across datasets, and that a classification head severely hurts generalization. To best our knowledge, this intuition has not been discussed in any of the prior works.

\subsection{Object Localization Network (OLN)}

The goal of OLN is to learn localization for objects and enable better generalization to new and unseen categories. OLN is a two-stage object proposer (see Figure 2). Similar to Faster R-CNN [45], OLN consists of a fullyconvolutional FCN stage and region-based RoI stage, but the key difference is that the classifiers in both FPN and ROI stages are replaced with localization quality predictions.

OLN-RPN. The input to this region proposal stage is the features from each level of ResNet feature pyramid [37]. Each feature map goes through a convolution layer followed by two separate layers, one for bounding box regression and the other for localization quality prediction. The network architecture design follows from the standard RPN heads.

We choose centerness [56] as the localization quality target and train both heads with L1 losses. Learning localization instead of classification at the proposal stage is crucial as it avoids overfitting to the foreground by classification. For training the localization quality estimation branch, we randomly sample 256 anchors having an IoU larger than 0.3 with the matched ground-truth boxes, without any explicit background sampling. For the box regression, we replace the standard box-delta targets $(x y h w)$ with distances from the location to four sides of the ground-truth box $(l r t b)$ as in [56]. We choose to use one anchor per feature location as opposed to 3 in RPN, because we observe its better generalization as each anchor can ingest more data.

OLN-Box. We take the top-scoring (e.g., well-centered) proposals from OLN-RPN and perform RoIAlign [25] to extract the region features from each feature pyramid level. Then we linearize each region features and feed it through two fc layers, followed by two separate fc layers, one for bounding box regression and the other for localization quality prediction. We use the same network architecture as Faster R-CNN heads [45]. We choose IoU as the localization quality target and train both heads with L1 losses. Learning localization quality at the second stage is integral as it allows the model to refine the proposal scoring and simultaneously avoid overfitting to the foreground. Compared to IoU-Net which requires manual proposal generation for IoU training, OLN directly computes the IoU targets from the OLN-RPN proposals and ground-truth boxes, thus greatly saving computation costs.

Extension - OLN-Mask. We explore whether more localization learning can further improve the generalization ability of our framework. To this end, we extend our OLNBox model to perform mask prediction by adding the classagnostic FCN mask head of Mask R-CNN, which we refer to as OLN-Mask model. Following our philosophy of OLN, and similarly to MS R-CNN [28], we learn to regress the IoU between the predicted and its GT mask.

Our mask-IoU predictor directly branches out from the fourth layer of the added FCN mask head, without having a feedback connection from the mask prediction unlike [28]. The IoU branch consists of a $3 \times 3$ convolution layer, a max pooling layer and three fully connected layers. During training, we assume mask annotations are available for the training categories, and use smooth-L1 loss for IoU regression.

Inference. During inference, the objectness score of a region $s$ is computed as a geometric mean of the centerness $c$ and IoU scores (box:b, mask:m) estimated by OLN-RPN and OLN-Box branches. For OLN-Box, the score $s=$ $\sqrt{(c \cdot b)}$. For OLN-Mask, the score is $s=\sqrt[3]{(c \cdot b \cdot m)}$.

\section{Experiments}

We study the generalization ability of the learned object proposal networks in three challenging generalization scenarios. We outperform all methods and strong baselines on them, and in some cases, significantly. First, we study 1) cross-category generalization on COCO dataset by evaluating average recall (AR) on new unseen classes. We compare with other state-of-the-art proposal and detection methods, and provide extensive ablation and analysis on OLN design. Also, we explore more challenging 2) open-world class-agnostic detection where the setup requires to detect all objects in an exhaustive and class-agnostic fashion. The testing images contain highly dissimilar objects to those in the training dataset; We train a model on COCO and test on RoboNet dataset [15] and evaluate the Average Precision (AP). We demonstrate more 3) cross-dataset generalization 

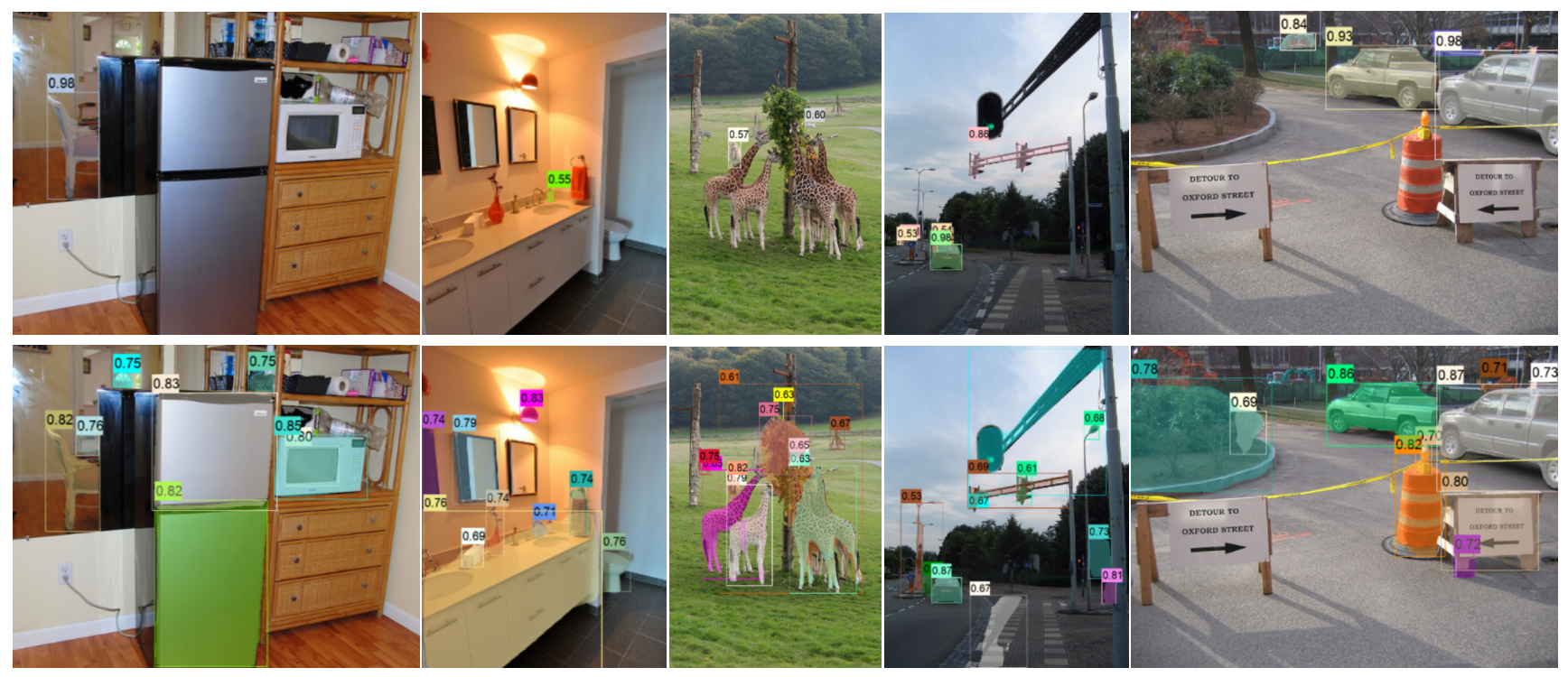

Figure 3: Visualization of $V O C \rightarrow$ Non-VOC category generalization. (Top: Mask R-CNN, Bottom: OLN-Mask).

results by testing on Object365 [48] and EpicKitchens [14] datasets. Finally, we study the 4) impact on long-tail object detection of OLN on LVIS [24] dataset. In the following, we provide experiment setups, evaluation protocol and results for each setting. In each $\S 4.1, \S 4.2, \S 4.3$, and $\S 4.4$, we use the same training and testing pipelines for all competing methods, for a fair comparison. All experiments use the same ResNet-50 [26] with feature pyramid [37] backbone, and the same box regression head and training hyperparameters as the Faster R-CNN [45], unless specified otherwise. More details are in supplementary materials.

\subsection{Cross-category generalization}

Setup. We split the COCO dataset into 20 seen (VOC) classes and 60 unseen (non-VOC) classes. We train a model with box annotations of only seen classes, and evaluate the recall on unseen non-VOC classes only. To avoid evaluating any recall on seen-class objects, we do not count those seenclass detection boxes into the budget $k$ when computing the Average Recall (AR@k) scores.

Comparison with other learning-based methods. We compare with learning-based single-stage and multi-stage methods. The comparison is in Table 1. For all methods, we use the standard official models available in MMDetection [11] and train with the same default $1 \times$ training and testing pipeline. Batch size of 16 ( 2 per GPU) and an initial learning rate of 0.02 are used. Note that the detection models are their class-agnostic versions with a binary classifier. The NMS threshold is set to 0.7 .

OLN-RPN improves the standard RPN [45] by a large margin of +5.2 AUC (+4.3 AR10 and +7.4 AR100), and outperforms all single-stage competitors. We also include RPN trained with 1-or-0 linear regression L1 loss instead of cross entropy loss and show that simply changing the loss function does not help. FCOS [56] with a binary classifier is a popular example of combining classification and localization cue (centerness). Two-stage OLN-Box shows a large gain of +4.9 AUC (+6.1 AR10 and +7.3 AR100) over Faster R-CNN. Guided anchoring (GA-RPN) [61] is an advanced iterative RPN with deformable convolution, and Cascade RPN [60] is the state-of-the-art RPN method with multi-stage refinement. OLN-Box outperforms all of them with a healthy margin: +3.3 AUC. In terms of model size, OLN-RPN is the same size as RPN, and OLN-Box is the same as Faster R-CNN.

Comparison with stronger baselines. We can come up with a stronger Faster R-CNN baseline where we do not suppress unseen classes in the classification. We filter out all unseen class objects to train Faster R-CNN *filtered* (BG sampling at $\mathrm{IoU}=0$ with unseen classes). Table 1 shows that OLN does better by learning to localize $(+3.0$ AUC), even when Faster R-CNN *filtered* has access to the ground truth boxes of novel objects.

It's worth noting that this paper's focus is on objectness learning for generalization of proposal models; we do not explore orthogonal factors such as advanced convolutions for feature alignment $[60,61]$ and the use of box regression statistics [60], that may further improve performance.

\footnotetext{
${ }^{1}$ We acknowledge that Pinheiro et al. use shallower network backbone (VGG16) than ours (ResNet-50). However, we show their reported numbers to provide a reference level. Both DeepMask20 and OLN-Box are trained on 20 VOC classes, and tested on all 80 classes.
} 


\begin{tabular}{l|l|c|ccccc}
\hline \multicolumn{2}{l|}{} & AUC & AR10 & AR30 & AR100 & AR300 & AR1k \\
\hline \hline Learning-free method & MCG [42] & 18.9 & 10.4 & 16.6 & 24.9 & 33.4 & 43.8 \\
\hline \multirow{4}{*}{ Single-stage models } & RPN [45] & 14.6 & 7.4 & 12.4 & 20.0 & 27.3 & 32.2 \\
& RPN w/ 1-or-0 reg. [45] & 14.6 & 7.5 & 13.1 & 19.5 & 27.2 & 32.4 \\
& FCOS [56] & 18.3 & 10.5 & 16.5 & 24.4 & 32.7 & 39.5 \\
& OLN-RPN (1-stage) & $\mathbf{1 9 . 8}$ & $\mathbf{1 1 . 7}$ & $\mathbf{1 9 . 4}$ & $\mathbf{2 7 . 4}$ & $\mathbf{3 3 . 7}$ & $\mathbf{3 9 . 7}$ \\
\hline \multirow{5}{*}{ Multi-stage models } & Faster R-CNN [45] & 19.4 & 11.7 & 17.4 & 25.5 & 34.1 & 42.2 \\
& Faster R-CNN *filtered* & 21.3 & 13.3 & 20.2 & 28.7 & 36.9 & 44.3 \\
& Guided Anchoring RPN [61] & 20.9 & 11.9 & 18.2 & 27.7 & 37.5 & $\mathbf{4 6 . 7}$ \\
& Cascade RPN [60] & 21.0 & 12.6 & 19.0 & 27.7 & 36.7 & 46.4 \\
& OLN-Box (2-stage) & $\mathbf{2 4 . 2}$ & $\mathbf{1 7 . 7}$ & $\mathbf{2 6 . 0}$ & $\mathbf{3 2 . 7}$ & $\mathbf{3 8 . 1}$ & 45.1 \\
\hline
\end{tabular}

Table 1: AR results of VOC $\rightarrow$ Non-VOC generalization.

\begin{tabular}{l|cccc}
\hline & AR10 & AR100 & AR1000 & AUC \\
\hline \hline EdgeBoxes [74] & 7.4 & 17.8 & 33.8 & 13.9 \\
Geodesic [32] & 4.0 & 18.0 & 35.9 & 12.6 \\
Sel.Search [59] & 5.2 & 16.3 & 35.7 & 12.6 \\
MCG [4] & 10.1 & 24.6 & 39.8 & 18.0 \\
\hline DeepMask20 [41] & 13.9 & 28.6 & 43.1 & 21.7 \\
\hline OLN-Box & $\mathbf{2 7 . 7}$ & $\mathbf{4 6 . 1}$ & $\mathbf{5 5 . 7}$ & $\mathbf{3 4 . 3}$ \\
\hline
\end{tabular}

Table 2: Comparison with learning-free methods on All Categories. We report average recall (AR) and AUC of learning free methods and ours vs DeepMask on VOC $\rightarrow$ All generalization. The scores of competing methods are taken from [41], which test object proposal methods on all 80 COCO classes.

Comparison with learning-free methods. We borrowed the DeepMask [41] setting that evaluates on 'all' categories in Table 2. To make a cleaner comparison, we report in Table 1 the MCG [42] (SOTA learning-free baseline) on 'unseen' split and find that OLN still maintains a healthy margin above it.

Ablation: modeling choices. We study what modeling component helps or hurts the generalization of OLN. We enumerate over different objectness cues, i.e., classification, centerness, IoU and Dice score, and their single-stage and two-stage configurations. The ablation is in Table 3 and Table 4.

How much does the second stage help? We notice that the second refinement head of two-stage models lead to better performance throughout all choices of objectness measure (Table 3 - $a$ vs $d-e, b$ vs $f-g$, and $c$ vs $h-j$ ). This can be attributed to better bounding box regression which has additional layers following the first stage, as also noted by He et al. [25]. This trend can be also seen between other single-stage $v s$ multi-stage methods in Table 1.

Impact of different objectness cues. We perform ablations on what objectness cue hurts or helps each stage of OLN. We start with single-stage models $(a-c)$ where the box regression pipeline is analogous to that of standard RPN except the transformed box coordinates. We compare

\begin{tabular}{|c|c|c|c|c|c|c|}
\hline & 1st-stage & 2nd-stage & AR10 & AR30 & AR100 & AR300 \\
\hline & \multicolumn{2}{|c|}{ Single-stage: } & & & & \\
\hline a. & Class & - & 7.8 & 12.8 & 20.0 & 27.9 \\
\hline b. & IoU & - & 9.7 & 15.8 & 22.2 & 28.3 \\
\hline \multirow[t]{2}{*}{ c. } & Center & - & 11.7 & 19.4 & 27.4 & 33.7 \\
\hline & \multicolumn{2}{|c|}{ Two-stage: } & & & & \\
\hline d. & Class $^{2}$ & Class & 11.9 & 17.6 & 25.5 & 34.4 \\
\hline e. & Class & Class & 12.0 & 17.8 & 25.6 & 34.3 \\
\hline f. & $\mathrm{IoU}$ & Center & 16.1 & 24.7 & 32.1 & 37.3 \\
\hline g. & IoU & IoU & 16.2 & 25.0 & 32.3 & 37.5 \\
\hline h. & Center & Center & 17.6 & 25.8 & 32.7 & 38.1 \\
\hline i. & Center & IoU & 17.7 & 26.0 & 32.7 & 38.1 \\
\hline j. & Center & Dice & 17.2 & 25.3 & 32.2 & 37.5 \\
\hline
\end{tabular}

Table 3: What objectness cues lead to better generalization? How much does the second stage help? Different OLN configurations on VOC $\rightarrow$ Non-VOC setting.

classification, centerness and IoU scores in Table 3; both localization-based scores outperform classification, and the centerness shows the best AR. Among the two-stage configurations, model- $(d)$ is comparable to Faster R-CNN, where only the last class score is used at testing. Again, we observe the overall superiority of localization-based objectness learning. We also notice that the second stage prefers IoU learning over centerness learning, whichever one of them is used in the first stage ( $f v s g$ and $h v s i$ ). Intuitively, IoU measure is sensitive to both location and shape of the detection, and thus better captures the quality of variablesized boxes in the second stage. On the other hand, centerness can be a more suitable measure for the first region proposal stage, where the shape of the anchors are fixed. Overall, the combination of centerness-then-IoU $(i)$ shows the best performance, implying their own contributions to the generalization. We also demonstrate that a different choice of localization quality also performs well $(j)$. For the rest of the paper, OLN-RPN and OLN-Box refer to model- $(c)$ and (i) respectively.

\footnotetext{
${ }^{2}$ For model- $(d)$, classification score from the first stage (RPN) is not used at inference; same as in the Faster R-CNN.
} 


\begin{tabular}{ll|cccc}
\hline 1st-stage & 2nd-stage & AR10 & AR30 & AR100 & AR300 \\
\hline \hline Center & - & $\mathbf{1 1 . 7}$ & $\mathbf{1 9 . 4}$ & $\mathbf{2 7 . 4}$ & $\mathbf{3 3 . 7}$ \\
Center + Class & - & 10.7 & 15.7 & 21.1 & 26.6 \\
\hline Center & IoU & $\mathbf{1 7 . 7}$ & $\mathbf{2 6 . 0}$ & $\mathbf{3 2 . 7}$ & $\mathbf{3 8 . 1}$ \\
Center & IoU + Class & 13.4 & 19.4 & 27.3 & 35.2 \\
Center + Class & IoU & 14.2 & 20.5 & 26.8 & 33.9 \\
Center + Class & IoU + Class & 13.3 & 18.9 & 26.5 & 33.8 \\
Center & Class & 13.0 & 18.7 & 26.5 & 34.6 \\
Class & Class & 12.0 & 17.8 & 25.6 & 34.3 \\
\hline
\end{tabular}

Table 4: How much does classifier hurt? Different OLN configurations on VOC $\rightarrow$ Non-VOC setting.
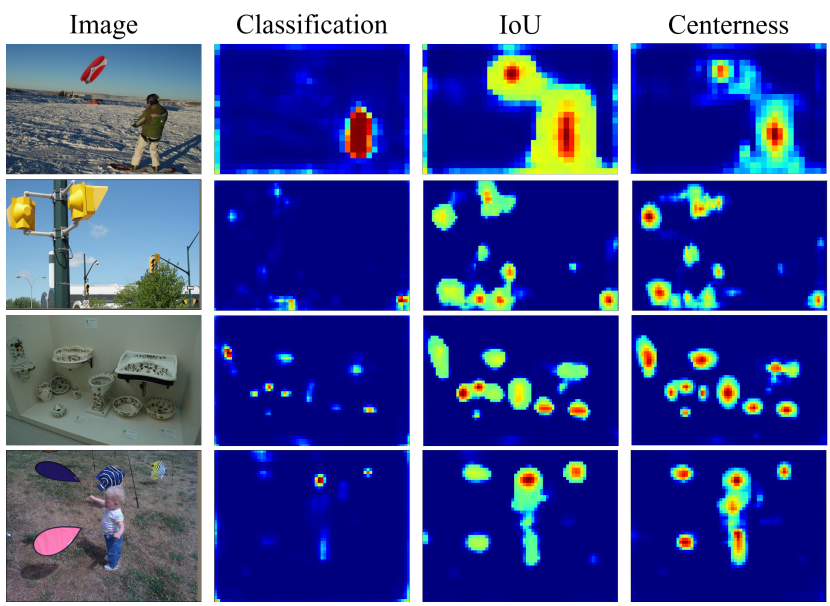

Figure 4: Visualization of score maps of classification, IoU and centerness, obtained from OLN-RPN stage.

How much does classifier hurt? Table 4 shows the results by adding a classifier branch to the best-performing OLN-RPN (c) and OLN-Box (i) models. Note that all the used objectness scores are geometrically averaged at test time. Throughout the single-stage and two-stage configurations, we observe a consistent drop in AR when adding a binary classifier. Largest drops are seen when having the classifiers in both stages. This validates our hypothesis that discriminative learning of object-or-not classification impedes generalization of object proposals, and that a pure localization-based objectness is the key to generalization.

Impact of positive / negative sampling ratio. Is the gain of OLN from the fact that we sample around the objects and avoid penalizing the unlabeled background objects? or does the permissive definition for positive helps generalization? In Table 5 we run Faster R-CNN with low sampling of background anchors similar to OLN. We validate that changing the sampling ratio does not help the baseline. This is because a balanced ratio (e.g., 1:1) of explicit negative vs positive samples is required for the binary classifier. Table 5 also shows various definition of positive / negative samples

\footnotetext{
${ }^{3}$ Default anchor sampling ratio of the Faster R-CNN.
}

\begin{tabular}{c|c|ccc|cc}
\hline & $\begin{array}{c}\text { B.G. } \\
\text { sample } \\
\text { ratio }\end{array}$ & $\begin{array}{c}\text { RPN } \\
\text { upper } \\
\text { thr. }\end{array}$ & $\begin{array}{c}\text { RPN } \\
\text { lower } \\
\text { thr. }\end{array}$ & $\begin{array}{c}\text { RoI } \\
\text { head } \\
\text { thr. }\end{array}$ & AR10 & AR100 \\
\hline \hline OLN-Box & $0 / 256$ & 0.3 & 0.1 & 0.3 & $\mathbf{1 7 . 7}$ & $\mathbf{3 2 . 7}$ \\
& $\mathbf{1 / 2 5 6}$ & 0.3 & 0.1 & 0.3 & $\mathbf{1 7 . 7}$ & 32.6 \\
\hline & $128 / 256^{3}$ & 0.7 & 0.3 & 0.5 & $\underline{11.7}$ & $\underline{25.5}$ \\
FR-CNN & $128 / 256$ & 0.3 & 0.1 & 0.3 & 7.3 & 17.8 \\
& $1 / 256$ & 0.7 & 0.3 & 0.5 & 10.1 & 21.8 \\
& $1 / 256$ & 0.3 & 0.1 & 0.3 & 6.7 & 14.9 \\
\hline \multirow{2}{*}{ FR-CNN } & $128 / 256$ & 0.7 & 0.3 & 0.5 & $\underline{13.1}$ & $\underline{28.4}$ \\
*filtered* $^{*}$ & $128 / 256$ & 0.3 & 0.1 & 0.3 & 8.3 & 18.8 \\
& $1 / 256$ & 0.7 & 0.3 & 0.5 & 12.0 & 25.8 \\
& $1 / 256$ & 0.3 & 0.1 & 0.3 & 7.9 & 16.5 \\
\hline
\end{tabular}

Table 5: Controlling B.G. sampling ratio and pos/neg definition.

\begin{tabular}{l|c|c|c}
\hline & mask IoU & box-AR10 & mask-AR10 \\
\hline \hline Mask-RCNN [25] & & 11.8 & 9.1 \\
\hline OLN-Box & N/A & 17.7 & - \\
OLN-Mask & & 17.9 & 15.8 \\
OLN-Mask & $\checkmark$ & 18.3 & 16.9 \\
\hline
\end{tabular}

Table 6: Does mask prediction learning help?

and that OLN-Box still outperforms Faster R-CNN and *filtered* models by a healthy margin. We think it is becuase OLN can learn more from the positives using localization cues instead of binary classification.

Does mask prediction learning help? In Table 6 we show the results for the mask-extended OLN model, OLNMask. We validate that incorporation of additional localization quality learning, i.e., mask-IoU, can further improve both the box-AR and mask-AR performances.

Visualization of VOC to Non-VOC transfer on COCO. We visualize class-agnostic Mask R-CNN and our OLNMask trained on VOC categories on COCO in Figure 3. The images come from COCO validation set. We observe Mask R-CNN tends to miss out-of-VOC class objects even when they appear in the dominant and salient region in an image, e.g., refrigerator (left column) and giraffes (right column). We believe this is because classification leads to learning just the union of $20 \mathrm{VOC}$ categories and use that knowledge to detect objectness. On the other hand, OLN can generalize well to detect many novel objects, such as fridge, microwave, vases, and giraffes, showing that localization leads to learning a more general notion of objectness.

Visualization of the learnt objectness map. In Figure 4, we visualize the confidence heatmap of different localization cues $v s$ classifier. We can see the IoU and centerness cues generalize better to novel objects than classification. For example, both IoU and centerness capture the flying kite, the traffic lights, handicrafts, the child and his/her toys, while the classifier head misses them. We use model- $a, b$, and $c$ in Table 3 for classification, IoU and centerness visualization respectively. 

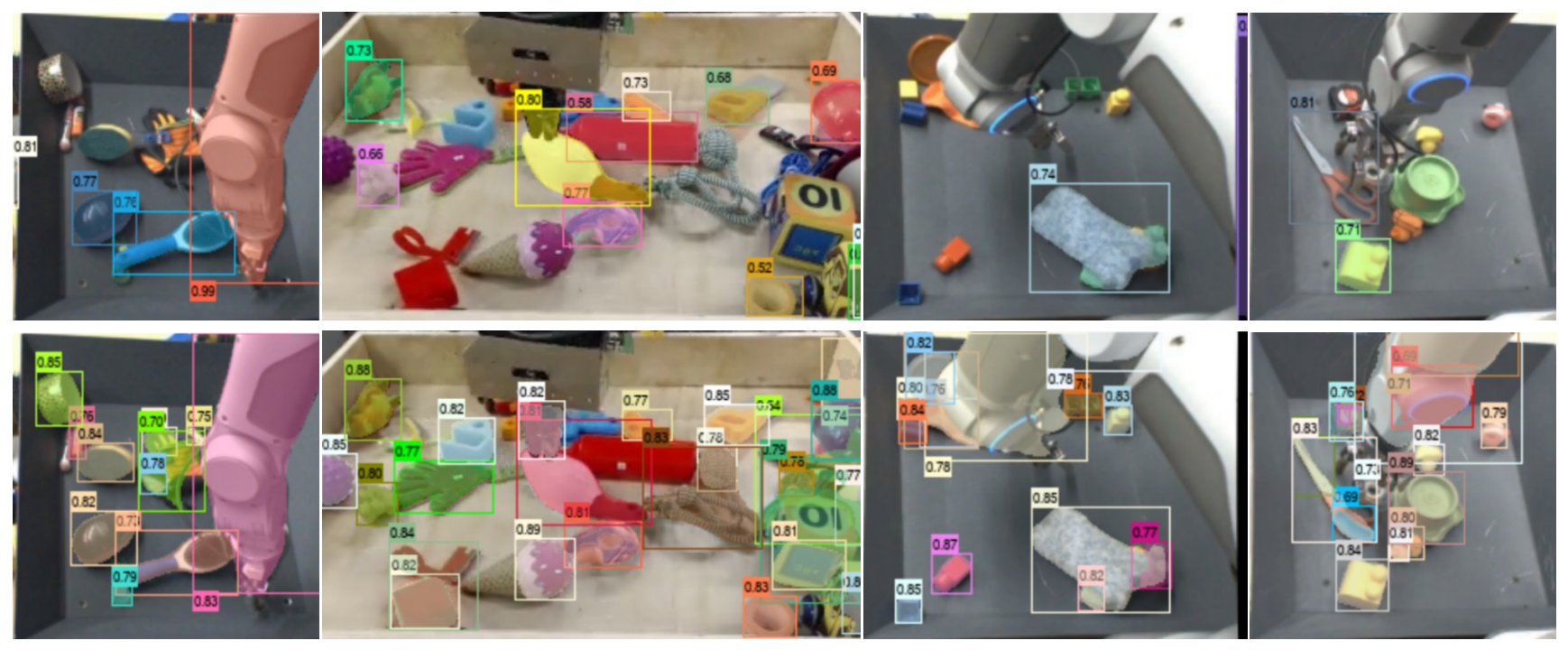

Figure 5: Visualization of COCO $\rightarrow$ RoboNet generalization. (Top: Mask R-CNN, Bottom: OLN-Mask.)

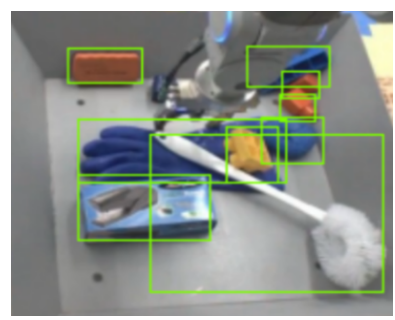

(a) GT boxes

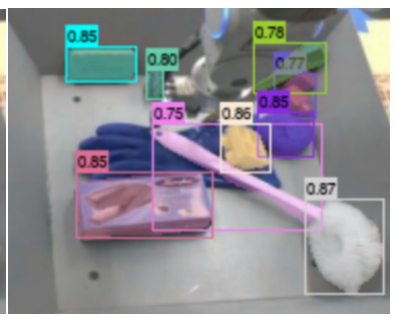

(b) OLN prediction

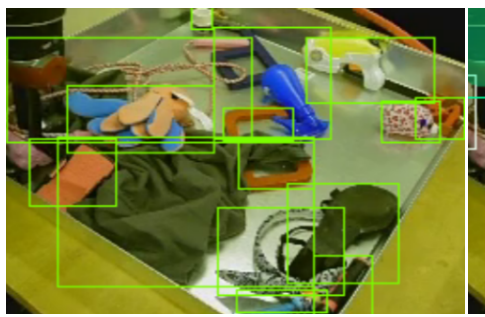

(c) GT boxes

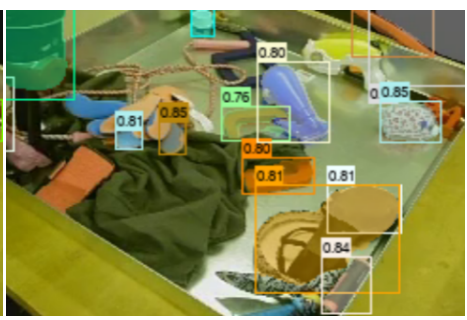

(d) OLN prediction

Figure 6: Analysis of failure modes of OLN on COCO $\rightarrow$ RoboNet generalization. For each sample, we show the ground-truth boxes on the left and our OLN-Mask predictions on the right.

\subsection{Open-World Class-Agnostic Detection}

Our ultimate goal is to learn a generalizable object detector that can detect every object in our open world. Taking a step towards this goal, we investigate the open-world, class-agnostic detection ability on highly diverse and hardto-name objects in RoboNet dataset. We train a model on COCO and test on held-out RoboNet [15] dataset.

Rigorous evaluation. Chavali et al. [9] report the vulnerability of current evaluation protocol for object proposals when evaluating on a partially-annotated dataset such as $\mathrm{COCO}$, because it fails to capture the performance (e.g., reward the recall) on all the non-COCO categories that are not annotated in the ground truth. To handle this issue, we exhaustively annotated bounding boxes for the presence of all objects in RoboNet images. This allows us to measure Average Precision as in standard object detection evaluation. Such evaluation captures whether a model can generate a few but highly-precise detections. We use NMS threshold of 0.5 and evaluate box-AP on top 100 proposals in this experiment.
Exhaustive box annotations. To enable the evaluation of our task also by the standard AP metric, we carefully annotated the RoboNet dataset for the presence of all objects in an exhaustive fashion. RoboNet is a large-scale robot manipulation video dataset collected for pre-training reinforcement learning [15]. The dataset contains very challenging clutter and object diversity that we find suitable for evaluating general objectness learning. Since the video data contains lots of redundancy at frame level, we resort to random sampling to construct a concise yet diverse evaluation set. Concretely, we randomly sampled 109 videos from the 162417 videos in the training set (roughly 1/1500) and for each video we select only the center frame. The parameters are picked by visually inspecting the diversity of resulting data (See Figure 6-(a, c) for examples). Our annotated dataset consists of total 109 frames, 1277 objects, and $34.8 \%$ of small objects, which are those with long side $<$ 32 pixels (same as $\mathrm{COCO}$ ). For object bounding box annotation, we adopt the method of labeling extreme points as proposed in [34], which results in better accuracy and effi- 


\begin{tabular}{l|cccccc}
\hline & $\mathrm{AP}$ & $\mathrm{AP}_{50}$ & $\mathrm{AP}_{75}$ & $\mathrm{AP}_{\mathrm{S}}$ & $\mathrm{AP}_{\mathrm{M}}$ & $\mathrm{AP}_{\mathrm{L}}$ \\
\hline \hline RPN [45] & 13.7 & 28.1 & 12.2 & 15.6 & 22.8 & 10.3 \\
Faster R-CNN [45] & 10.7 & 20.9 & 10.0 & 7.6 & 18.0 & 8.7 \\
Mask R-CNN [25] & 11.4 & 22.3 & 10.4 & 8.7 & 18.6 & 7.9 \\
\hline OLN-Box (Ours) & $\mathbf{2 4 . 6}$ & $\mathbf{4 7 . 1}$ & $\mathbf{2 3 . 0}$ & $\mathbf{2 0 . 9}$ & $\mathbf{3 2 . 1}$ & $\mathbf{1 5 . 4}$ \\
OLN-Mask (Ours) & $\mathbf{2 6 . 4}$ & $\mathbf{5 0 . 1}$ & $\mathbf{2 4 . 7}$ & $\mathbf{2 2 . 9}$ & $\mathbf{3 4 . 0}$ & $\mathbf{1 5 . 6}$ \\
\hline
\end{tabular}

Table 7: AP results of open-world class-agnostic detection in $\mathrm{COCO} \rightarrow$ RoboNet generalization.

ciency for the raters.

To exclude the background scene in the image which could contain hard-to-define objects/building structures, we label the object bin as well and crop out only the within-bin region for evaluation. This removes most of the ambiguity in objectness evaluation and allows us to have reasonably exhaustive annotation of objects for detection evaluation via average precision. To validate whether the size is large enough, we trained 5 independent runs of our model and observe a variance of $0.17 \mathrm{AP}$, showing that the results are quite reproducible on our dataset size.

Results. In this rigorous evaluation setting, larger gains are revealed for OLN models than in other experiments. In Table 7 we compare COCO $\rightarrow$ RoboNet generalization performance of OLN-Box and OLN-Mask models and class-agnostic Faster R-CNN and Mask R-CNN baselines. OLN models greatly outperform RPN and Faster RCNN by $\mathbf{+ 1 2 . 7}$ and $\mathbf{1 5 . 7}$ points AP, respectively. We also observe that the improvement in $\mathrm{AP}_{\mathrm{IoU}=50}$ is significantly larger, by $\mathbf{+ 2 2 . 0}$ and $\mathbf{2 9 . 1}$ points AP. These results provide strong evidence that OLN can achieve both high precision and recall in detecting novel objects, and that a few generated OLN proposals $(\sim 100)$ can be directly used as final bounding boxes for all objects in an image, i.e., classagnostic detection.

Visualization and failure cases. Figure 5 visualizes the box and mask predictions from baseline class-agnostic Mask R-CNN vs our OLN-Mask model. OLN is able to detect many novel objects (e.g. gloves, toys, tape, parts of toys), while the baseline misses most of them. Figure 6 presents an analysis of OLN failure modes by comparison with the ground-truth boxes. The false positive example is where OLN detects part of an object, e.g., it detects handle and head of a cleaning brush as two individual objects.

\subsection{More cross-dataset generalization}

We further study the generalization ability of OLN from one data source to different data sources.

\subsubsection{Generalization to Objects 365}

Objects 365 is a large-scale object detection dataset consisting of 365 object categories from our daily lives, which are a super-set of $80 \mathrm{COCO}$ categories. We train a model on

\begin{tabular}{l|cccc}
\hline & AR10 & AR20 & AR50 & AR100 \\
\hline \hline RPN [45] & 8.8 & 12.5 & 18.1 & 22.5 \\
Faster R-CNN [45] & 10.1 & 13.7 & 19.1 & 23.1 \\
Mask R-CNN [25] & 10.1 & 13.7 & 19.2 & 23.3 \\
\hline OLN-Box (Ours) & $\mathbf{1 3 . 1}$ & $\mathbf{1 8 . 8}$ & $\mathbf{2 6 . 0}$ & $\mathbf{3 0 . 6}$ \\
OLN-Mask (Ours) & $\mathbf{1 3 . 3}$ & $\mathbf{1 8 . 9}$ & $\mathbf{2 6 . 0}$ & $\mathbf{3 0 . 7}$ \\
\hline
\end{tabular}

Table 8: AR results of $\mathrm{COCO} \rightarrow$ Objects365 generalization.

COCO and test on non-overlapping classes on this dataset. We follow the same AR@k evaluation protocol in Section 4.1, treating all the 365 classes as a single 'object' class, and leaving out the detection boxes on seen COCO 80 classes when evaluating top-k recall.

Results. Table 8 shows the COCO $\rightarrow$ Objects 365 generalization results. OLN outperforms all the classificationbased baselines for all number of proposals considered. Especially, the gains over the RPN and Faster R-CNN start from +4.5 and 3.3 AR10 and achieve large gaps of +8.2 and 7.6 points in AR100.

\subsubsection{Generalization to EpicKitchens}

EpicKitchens is the largest egocentric vision dataset containing the wearers' different kitchen activities like washing and cooking, with many different objects like food, kitchenware and appliances. It provides the automatic mask annotations of 66M objects, which they extracted by using the off-the-shelf Mask R-CNN [25] trained on COCO dataset. Therefore, we use their official mask annotations as our baseline in this experiment.

Results. AR or AP are difficult to compute on EpicKitchens because there are no groundtruth boxes or masks. Therefore, we visually compare the EpicKitchens mask annotations and our OLN-Mask prediction outputs in Figure 1 and 7, which are randomly selected examples. We observe that OLN-Mask is able to detect almost complete set of objects in an image, while the baseline Mask R-CNN misses most out-of-sample objects. To illustrate, OLN is able to detect $a$ stack of dishes, dough balls, a toaster, half side of a microwave and its front window, as shown in Figure 1 and 7. These are novel categories outside of the COCO vocabulary, and appear in different configurations and viewpoints rarely seen in COCO. This demonstrate the clear benefits of learning localization for novel object detection.

\subsection{Impact on long-tail object detection}

Large vocabulary detection has gained much attention lately in research community [22, 24, 52, 65] because the problem captures the Zipf's law of visual world, i.e. most categories appear with low frequency. We want to see whether OLN can aid downstream detection in a challenging long-tail setting, on LVIS dataset [24], e.g., only 1-10 

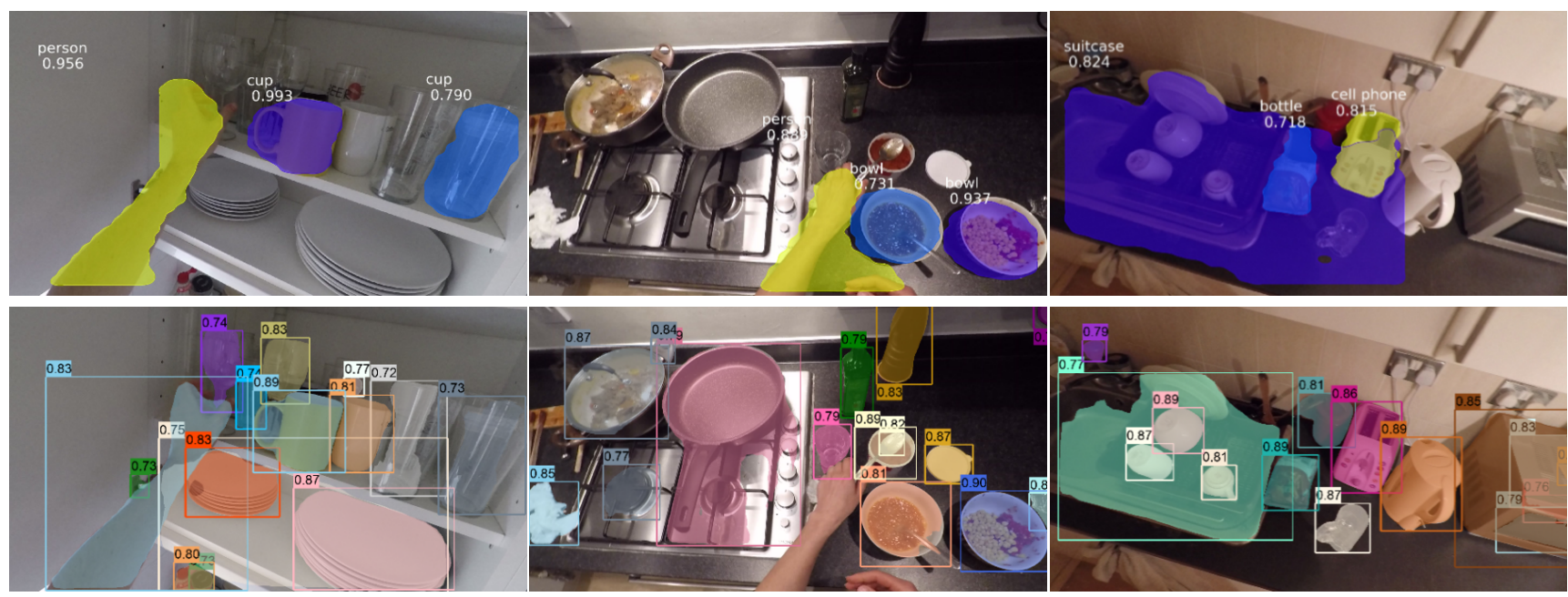

Figure 7: Visualization of $\mathrm{COCO} \rightarrow$ EpicKitchens generalization. We compare the EpicKitchen's official annotations [14] obtained from Mask R-CNN (left) vs. ours from OLN-Mask (right).

\begin{tabular}{|c|c|c|c|c|c|c|c|c|c|}
\hline Detector & Proposals & AR & AR rare & AR comm. & AR freq. & $\mathrm{AP}$ & AP rare & AP comm. & AP freq. \\
\hline & RPN [45] & 32.3 & 14.6 & 30.2 & 41.9 & 24.1 & 13.0 & 23.7 & 28.9 \\
\hline FR-CNN & OLN-RPN & $33.8(+1.5)$ & $19.9(+5.3)$ & $32.1(+1.9)$ & $41.4(-0.5)$ & $25.5(+1.4)$ & $16.4(+3.4)$ & $25.4(+1.8)$ & $29.3(+0.4)$ \\
\hline
\end{tabular}

Table 9: Long-tail detection on LVIS v0.5 using Faster R-CNN [45].
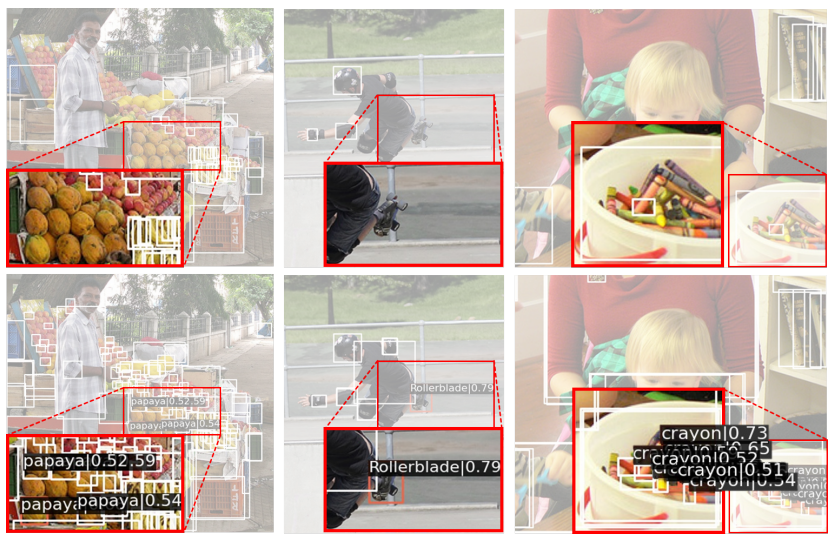

Figure 8: Visualization of long-tail detection on LVIS using Faster R-CNN. (Top: RPN, Bottom: OLN-RPN.)

training samples are available for rare categories. Table 9 shows that a drop-in replacement of RPN [45] with OLNRPN helps the overall average recall $(\mathrm{AR})$ by +1.5 , where most gains come from rare $(\mathbf{+ 5 . 3})$ and common categories $(+1.9)$. The 5-point gain in rare categories recall demonstrates that OLN can benefit the long-tail of large vocabulary detection. In fact, this gain transfers quite well to overall average precision (AP) improvement +1.4 , where most gains come from the rare $(+\mathbf{3 . 4})$ and common $(+1.8)$ categories. We choose $k=300$ detections per image, following the convention of the community. Our baseline matches the performance of Faster R-CNN reported by [67] in the same setting.

Figure 8 presents a few challenging cases of long-tail detection on rare categories - piles of papaya, crayon, and a small roller-blade. Compared to the baseline, a drop-in OLN-RPN replacement enables better detection on these challenging cases. We can see many missed papayas, crayons and the small roller-blade are now correctly localized and classified.

\section{Conclusion}

In this paper we tackle the challenging problem of learning novel object proposals. Observing the tendency of existing proposals to overfit to training categories, we propose a simple yet effective framework (OLN) that learns to propose novel objects by learning localization cues (centerness, IoU, and regression) instead of binary classification. Experiments show that OLN outperforms existing methods on cross-category generalization on COCO, as well as crossdataset settings on RoboNet, Object365, and EpicKitchens. Moreover, a drop-in replacement of OLN improves the performance on large vocabulary and egocentric video object detection on LVIS dataset. We believe OLN is a step forward in open-world novel object understanding with many applications. 


\section{References}

[1] Robust Vision Challenge 2020. Robust vision challenge 2020 official website. http://www.robustvision. net /, 2020.

[2] Bogdan Alexe, Thomas Deselaers, and Vittorio Ferrari. Measuring the objectness of image windows. IEEE transactions on pattern analysis and machine intelligence, 34(11):21892202, 2012.

[3] Pablo Arbeláez, Bharath Hariharan, Chunhui Gu, Saurabh Gupta, Lubomir Bourdev, and Jitendra Malik. Semantic segmentation using regions and parts. In 2012 IEEE conference on computer vision and pattern recognition, pages 33783385. IEEE, 2012.

[4] Pablo Arbeláez, Jordi Pont-Tuset, Jonathan T Barron, Ferran Marques, and Jitendra Malik. Multiscale combinatorial grouping. In Proceedings of the IEEE conference on computer vision and pattern recognition, pages 328-335, 2014.

[5] Aditya Arun, CV Jawahar, and M Pawan Kumar. Dissimilarity coefficient based weakly supervised object detection. In Proceedings of the IEEE/CVF Conference on Computer Vision and Pattern Recognition, pages 9432-9441, 2019.

[6] Ankan Bansal, Karan Sikka, Gaurav Sharma, Rama Chellappa, and Ajay Divakaran. Zero-shot object detection. In Proceedings of the European Conference on Computer Vision (ECCV), pages 384-400, 2018.

[7] Hakan Bilen and Andrea Vedaldi. Weakly supervised deep detection networks. In Proceedings of the IEEE Conference on Computer Vision and Pattern Recognition, pages 28462854, 2016.

[8] Joao Carreira, Rui Caseiro, Jorge Batista, and Cristian Sminchisescu. Semantic segmentation with second-order pooling. In European Conference on Computer Vision, pages 430443. Springer, 2012.

[9] Neelima Chavali, Harsh Agrawal, Aroma Mahendru, and Dhruv Batra. Object-proposal evaluation protocol is' gameable'. In Proceedings of the IEEE conference on computer vision and pattern recognition, pages 835-844, 2016.

[10] Hao Chen, Yali Wang, Guoyou Wang, and Yu Qiao. Lstd: A low-shot transfer detector for object detection. In Proceedings of the AAAI Conference on Artificial Intelligence, volume 32, 2018.

[11] Kai Chen, Jiaqi Wang, Jiangmiao Pang, Yuhang Cao, Yu Xiong, Xiaoxiao Li, Shuyang Sun, Wansen Feng, Ziwei Liu, Jiarui Xu, Zheng Zhang, Dazhi Cheng, Chenchen Zhu, Tianheng Cheng, Qijie Zhao, Buyu Li, Xin Lu, Rui Zhu, Yue Wu, Jifeng Dai, Jingdong Wang, Jianping Shi, Wanli Ouyang, Chen Change Loy, and Dahua Lin. MMDetection: Open mmlab detection toolbox and benchmark. arXiv preprint arXiv:1906.07155, 2019.

[12] Minsu Cho, Suha Kwak, Cordelia Schmid, and Jean Ponce. Unsupervised object discovery and localization in the wild: Part-based matching with bottom-up region proposals. In Proceedings of the IEEE conference on computer vision and pattern recognition, pages 1201-1210, 2015.

[13] Jifeng Dai, Kaiming He, and Jian Sun. Convolutional feature masking for joint object and stuff segmentation. In Proceed- ings of the IEEE conference on computer vision and pattern recognition, pages 3992-4000, 2015.

[14] Dima Damen, Hazel Doughty, Giovanni Maria Farinella, Sanja Fidler, Antonino Furnari, Evangelos Kazakos, Davide Moltisanti, Jonathan Munro, Toby Perrett, Will Price, and Michael Wray. The epic-kitchens dataset: Collection, challenges and baselines. IEEE Transactions on Pattern Analysis and Machine Intelligence (TPAMI), 2020.

[15] Sudeep Dasari, Frederik Ebert, Stephen Tian, Suraj Nair, Bernadette Bucher, Karl Schmeckpeper, Siddharth Singh, Sergey Levine, and Chelsea Finn. Robonet: Large-scale multi-robot learning. arXiv preprint arXiv:1910.11215, 2019.

[16] Thomas Deselaers, Bogdan Alexe, and Vittorio Ferrari. Weakly supervised localization and learning with generic knowledge. International journal of computer vision, 100(3):275-293, 2012.

[17] Ali Diba, Vivek Sharma, Ali Pazandeh, Hamed Pirsiavash, and Luc Van Gool. Weakly supervised cascaded convolutional networks. In Proceedings of the IEEE conference on computer vision and pattern recognition, pages 914-922, 2017.

[18] Ian Endres and Derek Hoiem. Category-independent object proposals with diverse ranking. IEEE transactions on pattern analysis and machine intelligence, 36(2):222-234, 2013.

[19] M. Everingham, S. M. A. Eslami, L. Van Gool, C. K. I. Williams, J. Winn, and A. Zisserman. The pascal visual object classes challenge: A retrospective. International Journal of Computer Vision, 111(1):98-136, Jan. 2015.

[20] Andrea Frome, Greg Corrado, Jonathon Shlens, Samy Bengio, Jeffrey Dean, Marc'Aurelio Ranzato, and Tomas Mikolov. Devise: A deep visual-semantic embedding model. 2013.

[21] Yan Gao, Boxiao Liu, Nan Guo, Xiaochun Ye, Fang Wan, Haihang You, and Dongrui Fan. C-midn: Coupled multiple instance detection network with segmentation guidance for weakly supervised object detection. In Proceedings of the IEEE/CVF International Conference on Computer Vision, pages 9834-9843, 2019.

[22] Golnaz Ghiasi, Yin Cui, Aravind Srinivas, Rui Qian, TsungYi Lin, Ekin D Cubuk, Quoc V Le, and Barret Zoph. Simple copy-paste is a strong data augmentation method for instance segmentation. arXiv preprint arXiv:2012.07177, 2020.

[23] Spyros Gidaris and Nikos Komodakis. Attend refine repeat: Active box proposal generation via in-out localization. arXiv preprint arXiv:1606.04446, 2016.

[24] Agrim Gupta, Piotr Dollar, and Ross Girshick. LVIS: A dataset for large vocabulary instance segmentation. In Proceedings of the IEEE Conference on Computer Vision and Pattern Recognition, 2019.

[25] Kaiming He, Georgia Gkioxari, Piotr Dollár, and Ross Girshick. Mask r-cnn. In Proceedings of the IEEE international conference on computer vision, pages 2961-2969, 2017.

[26] Kaiming He, Xiangyu Zhang, Shaoqing Ren, and Jian Sun. Deep residual learning for image recognition. In Proceedings of the IEEE conference on computer vision and pattern recognition, pages 770-778, 2016. 
[27] Judy Hoffman, Sergio Guadarrama, Eric Tzeng, Ronghang $\mathrm{Hu}$, Jeff Donahue, Ross Girshick, Trevor Darrell, and Kate Saenko. Lsda: Large scale detection through adaptation. arXiv preprint arXiv:1407.5035, 2014.

[28] Zhaojin Huang, Lichao Huang, Yongchao Gong, Chang Huang, and Xinggang Wang. Mask scoring r-cnn. In Proceedings of the IEEE conference on computer vision and pattern recognition, pages 6409-6418, 2019.

[29] Borui Jiang, Ruixuan Luo, Jiayuan Mao, Tete Xiao, and Yuning Jiang. Acquisition of localization confidence for accurate object detection. In Proceedings of the European Conference on Computer Vision (ECCV), pages 784-799, 2018.

[30] KJ Joseph, Salman Khan, Fahad Shahbaz Khan, and Vineeth N Balasubramanian. Towards open world object detection. In Proceedings of the IEEE/CVF Conference on Computer Vision and Pattern Recognition, pages 5830-5840, 2021.

[31] Bingyi Kang, Zhuang Liu, Xin Wang, Fisher Yu, Jiashi Feng, and Trevor Darrell. Few-shot object detection via feature reweighting. In Proceedings of the IEEE/CVF International Conference on Computer Vision, pages 8420-8429, 2019.

[32] Philipp Krähenbühl and Vladlen Koltun. Geodesic object proposals. In European conference on computer vision, pages 725-739. Springer, 2014.

[33] Weicheng Kuo, Bharath Hariharan, and Jitendra Malik. Deepbox: Learning objectness with convolutional networks. In Proceedings of the IEEE international conference on computer vision, pages 2479-2487, 2015.

[34] Alina Kuznetsova, Hassan Rom, Neil Alldrin, Jasper Uijlings, Ivan Krasin, Jordi Pont-Tuset, Shahab Kamali, Stefan Popov, Matteo Malloci, Tom Duerig, et al. The open images dataset v4: Unified image classification, object detection, and visual relationship detection at scale. arXiv preprint arXiv:1811.00982, 2018.

[35] Suha Kwak, Minsu Cho, Ivan Laptev, Jean Ponce, and Cordelia Schmid. Unsupervised object discovery and tracking in video collections. In Proceedings of the IEEE international conference on computer vision, pages 3173-3181, 2015.

[36] Hongyang Li, Yu Liu, Wanli Ouyang, and Xiaogang Wang. Zoom out-and-in network with map attention decision for region proposal and object detection. International Journal of Computer Vision, 127(3):225-238, 2019.

[37] Tsung-Yi Lin, Piotr Dollár, Ross Girshick, Kaiming He, Bharath Hariharan, and Serge Belongie. Feature pyramid networks for object detection. In Proceedings of the IEEE conference on computer vision and pattern recognition, pages 2117-2125, 2017.

[38] Tsung-Yi Lin, Michael Maire, Serge Belongie, James Hays, Pietro Perona, Deva Ramanan, Piotr Dollár, and C Lawrence Zitnick. Microsoft coco: Common objects in context. In European conference on computer vision, pages 740-755. Springer, 2014.

[39] Santiago Manen, Matthieu Guillaumin, and Luc Van Gool. Prime object proposals with randomized prim's algorithm. In Proceedings of the IEEE international conference on computer vision, pages 2536-2543, 2013.
[40] Fausto Milletari, Nassir Navab, and Seyed-Ahmad Ahmadi. V-net: Fully convolutional neural networks for volumetric medical image segmentation. In 2016 fourth international conference on $3 D$ vision (3DV), pages 565-571. IEEE, 2016.

[41] Pedro OO Pinheiro, Ronan Collobert, and Piotr Dollár. Learning to segment object candidates. In Advances in Neural Information Processing Systems, pages 1990-1998, 2015.

[42] Jordi Pont-Tuset, Pablo Arbelaez, Jonathan T Barron, Ferran Marques, and Jitendra Malik. Multiscale combinatorial grouping for image segmentation and object proposal generation. IEEE transactions on pattern analysis and machine intelligence, 39(1):128-140, 2016.

[43] Shafin Rahman, Salman Khan, and Fatih Porikli. Zero-shot object detection: Learning to simultaneously recognize and localize novel concepts. In Asian Conference on Computer Vision, pages 547-563. Springer, 2018.

[44] Joseph Redmon and Ali Farhadi. Yolo9000: better, faster, stronger. In Proceedings of the IEEE conference on computer vision and pattern recognition, pages 7263-7271, 2017.

[45] Shaoqing Ren, Kaiming He, Ross Girshick, and Jian Sun. Faster r-cnn: Towards real-time object detection with region proposal networks. In C. Cortes, N. Lawrence, D. Lee, M. Sugiyama, and R. Garnett, editors, Advances in Neural Information Processing Systems, volume 28, pages 91-99. Curran Associates, Inc., 2015.

[46] Hamid Rezatofighi, Nathan Tsoi, JunYoung Gwak, Amir Sadeghian, Ian Reid, and Silvio Savarese. Generalized intersection over union: A metric and a loss for bounding box regression. In Proceedings of the IEEE/CVF Conference on Computer Vision and Pattern Recognition, pages 658-666, 2019.

[47] Michael Rubinstein, Armand Joulin, Johannes Kopf, and Ce Liu. Unsupervised joint object discovery and segmentation in internet images. In Proceedings of the IEEE conference on computer vision and pattern recognition, pages 1939-1946, 2013.

[48] Shuai Shao, Zeming Li, Tianyuan Zhang, Chao Peng, Gang Yu, Xiangyu Zhang, Jing Li, and Jian Sun. Objects365: A large-scale, high-quality dataset for object detection. In Proceedings of the IEEE international conference on computer vision, pages 8430-8439, 2019.

[49] Bharat Singh, Hengduo Li, Abhishek Sharma, and Larry S Davis. R-fcn-3000 at 30fps: Decoupling detection and classification. In Proceedings of the IEEE Conference on Computer Vision and Pattern Recognition, pages 1081-1090, 2018.

[50] Krishna Kumar Singh, Santosh Divvala, Ali Farhadi, and Yong Jae Lee. Dock: Detecting objects by transferring common-sense knowledge. In Proceedings of the European Conference on Computer Vision (ECCV), pages 492-508, 2018.

[51] Jin Sun and Haibin Ling. Scale and object aware image retargeting for thumbnail browsing. In 2011 International Conference on Computer Vision, pages 1511-1518. IEEE, 2011.

[52] Jingru Tan, Gang Zhang, Hanming Deng, Changbao Wang, Lewei Lu, Quanquan Li, and Jifeng Dai. 1st place solution 
of lvis challenge 2020: A good box is not a guarantee of a good mask. arXiv preprint arXiv:2009.01559, 2020.

[53] Peng Tang, Xinggang Wang, Song Bai, Wei Shen, Xiang Bai, Wenyu Liu, and Alan Yuille. Pcl: Proposal cluster learning for weakly supervised object detection. IEEE transactions on pattern analysis and machine intelligence, 42(1):176191, 2018

[54] Peng Tang, Xinggang Wang, Xiang Bai, and Wenyu Liu. Multiple instance detection network with online instance classifier refinement. In Proceedings of the IEEE Conference on Computer Vision and Pattern Recognition, pages 28432851, 2017.

[55] Yuxing Tang, Josiah Wang, Boyang Gao, Emmanuel Dellandréa, Robert Gaizauskas, and Liming Chen. Large scale semi-supervised object detection using visual and semantic knowledge transfer. In Proceedings of the IEEE Conference on Computer Vision and Pattern Recognition, pages 21192128, 2016.

[56] Zhi Tian, Chunhua Shen, Hao Chen, and Tong He. Fcos: Fully convolutional one-stage object detection. In Proceedings of the IEEE international conference on computer vision, pages 9627-9636, 2019.

[57] Lachlan Tychsen-Smith and Lars Petersson. Improving object localization with fitness nms and bounded iou loss. In Proceedings of the IEEE conference on computer vision and pattern recognition, pages 6877-6885, 2018.

[58] Jasper Uijlings, Stefan Popov, and Vittorio Ferrari. Revisiting knowledge transfer for training object class detectors. In Proceedings of the IEEE Conference on Computer Vision and Pattern Recognition, pages 1101-1110, 2018.

[59] Jasper RR Uijlings, Koen EA Van De Sande, Theo Gevers, and Arnold WM Smeulders. Selective search for object recognition. International journal of computer vision, 104(2):154-171, 2013.

[60] Thang Vu, Hyunjun Jang, Trung X Pham, and Chang D Yoo. Cascade rpn: Delving into high-quality region proposal network with adaptive convolution. arXiv preprint arXiv:1909.06720, 2019.

[61] Jiaqi Wang, Kai Chen, Shuo Yang, Chen Change Loy, and Dahua Lin. Region proposal by guided anchoring. In Proceedings of the IEEE/CVF Conference on Computer Vision and Pattern Recognition, pages 2965-2974, 2019.

[62] Jiajie Wang, Jiangchao Yao, Ya Zhang, and Rui Zhang. Collaborative learning for weakly supervised object detection. arXiv preprint arXiv:1802.03531, 2018.

[63] Naiyan Wang, Siyi Li, Abhinav Gupta, and Dit-Yan Yeung. Transferring rich feature hierarchies for robust visual tracking. arXiv preprint arXiv:1501.04587, 2015.

[64] Rui Wang, Dhruv Mahajan, and Vignesh Ramanathan. What leads to generalization of object proposals? In European Conference on Computer Vision, pages 464-478. Springer, 2020.

[65] Tao Wang, Yu Li, Bingyi Kang, Junnan Li, Junhao Liew, Sheng Tang, Steven Hoi, and Jiashi Feng. The devil is in classification: A simple framework for long-tail instance segmentation. In European conference on computer vision, pages 728-744. Springer, 2020.
[66] Weiyao Wang, Matt Feiszli, Heng Wang, and Du Tran. Unidentified video objects: A benchmark for dense, openworld segmentation. arXiv preprint arXiv:2104.04691, 2021.

[67] Xin Wang, Thomas E Huang, Trevor Darrell, Joseph E Gonzalez, and Fisher Yu. Frustratingly simple few-shot object detection. arXiv preprint arXiv:2003.06957, 2020.

[68] Yu-Xiong Wang, Deva Ramanan, and Martial Hebert. Metalearning to detect rare objects. In Proceedings of the IEEE/CVF International Conference on Computer Vision, pages 9925-9934, 2019.

[69] Shengkai Wu, Xiaoping Li, and Xinggang Wang. Iou-aware single-stage object detector for accurate localization. Image and Vision Computing, 97:103911, 2020.

[70] Yongqin Xian, Christoph H Lampert, Bernt Schiele, and Zeynep Akata. Zero-shot learning-a comprehensive evaluation of the good, the bad and the ugly. IEEE transactions on pattern analysis and machine intelligence, 41(9):22512265, 2018.

[71] Xiaopeng Yan, Ziliang Chen, Anni Xu, Xiaoxi Wang, Xiaodan Liang, and Liang Lin. Meta r-cnn: Towards general solver for instance-level low-shot learning. In Proceedings of the IEEE/CVF International Conference on Computer Vision, pages 9577-9586, 2019.

[72] Bin Yang, Junjie Yan, Zhen Lei, and Stan Z Li. Craft objects from images. In Proceedings of the IEEE Conference on Computer Vision and Pattern Recognition, pages 6043 6051, 2016.

[73] Hao Yang, Hao Wu, and Hao Chen. Detecting 11k classes: Large scale object detection without fine-grained bounding boxes. In Proceedings of the IEEE/CVF International Conference on Computer Vision, pages 9805-9813, 2019.

[74] C Lawrence Zitnick and Piotr Dollár. Edge boxes: Locating object proposals from edges. In European conference on computer vision, pages 391-405. Springer, 2014. 